\title{
Research Progress of Treating Diabetic Nephropathy with Traditional Chinese Medicine
}

\author{
Ningjing $\mathrm{Wu}^{1}$, Rong $\mathrm{Li}^{1}$, Zongliang Song2* \\ 1Shaanxi University of Traditional Chinese Medicine, Xianyang 712000, Shaanxi Province, China \\ ${ }^{2}$ The Affiliated Hospital of Shaanxi University of Traditional Chinese Medicine, Xianyang 712000, Shaanxi Province, China \\ *Corresponding author: Zongliang Song, songzongliang@163.com

\begin{abstract}
By collecting and sorting out the literature and journals on the clinical use of Chinese medicine in the treatment of diabetic nephropathy in recent years, it is concluded that Chinese medicine is effective in the treatment of DKD. Chinese medicine therapy has a unique role in the treatment of diabetic nephropathy. How to correct syndrome differentiation and treatment? Improving clinical effects is the research direction that all medical staff will work together in the future.
\end{abstract}

Keywords: Diabetic nephropathy; Dialectical treatment; Traditional Chinese medicine therapy

Publication date: July 2021; Online publication: July 31, 2021

\section{Introduction}

Diabetic nephropathy (DKD) refers to patients with DM who have multiple, minimal or large amounts of proteinuria within three to six months on the premise of excluding kidney damage caused by other causes. Its clinical manifestations include varying degrees of proteinuria, renal impairment, hypertension, and edema. At this stage, the prevalence and morbidity of DKD in my country remain high. However, the treatment plan of modern medicine is alternative therapy, and the effect is not satisfactory. Additionally, there are a lot of complications and adverse reactions. Studies by modern scholars have shown that traditional Chinese medicine (TCM) has unique advantages in treating and improving the clinical symptoms of patients with DKD and protecting kidney function.

\section{The etiology and pathogenesis of DKD}

Based on the understanding of the overall concept, the onset of Diabetes is the kidney, which is mostly related to the liver and spleen, and the heart and stomach are often involved in the late stage. The main causes of the disease are mostly lack of endowment, overwork, imbalanced diet, emotional depression and other factors. Under the influence of long-term different factors, it causes kidney damage, which leads to the breakdown of homeostasis and the development of the disease. Diabetes nephropathy is one of the complications of the late stage of Diabetes disease. After a long period of illness, patients suffer severe loss of Qi and Yin, Yin is damaged and Yang, which will eventually lead to the consequences of both yin and yang deficiency. If the blood stasis does not go away, the new blood does not grow, thus depleting the body's blood and body fluid. In short, Diabetes nephropathy is a disease in which Yin deficiency is the root, dryness and heat are the target, and deficiency and excess are mixed.

\section{Syndrome differentiation and treatment of DKD}

Today's famous Chinese medicine experts have their own opinions on the differentiation of DKD. Bing Chenet al. ${ }^{[1]}$ based on the dialectics of Yin and yang of the internal organs, and divided the disease into 
five types: those with deficiency of Qi and Yin are mainly used to replenish Qi and nourish Yin; To invigorate the spleen and reduce dampness. Syndrome of liver and kidney yin deficiency, the treatment is to replenish liver and kidney; Yin and Yang deficiency, mainly warming yang to invigorate the kidney and invigorating Qi; Qi and blood deficiency syndrome, mainly tonifying the kidney, strengthening the spleen, aroma-reducing turbidity, and promoting blood circulation and removing blood stasis. Yiping Chen ${ }^{\text {[2] }}$ divided the disease into three types according to different clinical symptoms. The treatment of deficiency of both Qi and Yin is mainly to replenish Qi and nourish Yin, the treatment of deficiency of spleen and kidney combined with Qi deficiency and blood stasis is mainly to invigorate the spleen and kidney. Replenishing Qi and activating blood; The main treatment is to warm the kidney and dilute water, dissipate blood stasis and relieve turbidity.

\section{DKD common single Chinese medicine and Chinese medicine extract}

The pharmacology of TCM follows the continuous development of modern medicine, and the efficacy of single-flavored Chinese medicine for DKD has received more attention.

\subsection{Astragalus}

Astragalus ${ }^{[3]}$ has the effects of invigorating Qi, diuresis and reducing swelling. According to clinical data in recent years, the urine microalbumin of patients taking Astragalus membranaceus was significantly lower than that of patients who did not take the medicine. This indicates that Astragalus membranaceus can effectively reduce urine protein and delay and block the development of DKD. According to relevant data, the astragalus polysaccharide contained in astragalus can improve the renal function of patients with DM and has a certain effect on protecting the kidneys.

\subsection{Rhubarb}

Rhubarb has the functions of detoxifying heat, removing congestion, and dredging the meridians.

Scientific research has shown that certain components contained in rhubarb have the effects of reducing azotemia, inhibiting compensatory hypertrophy of residual renal tissue, correcting lipid metabolism disorders, reducing proteinuria, improving microcirculation, anticoagulation, antithrombosis, etc., and delaying DKD. Disease development process.

\subsection{Pueraria lobata}

Pueraria lobata ${ }^{[4]}$ has the effect of producing body fluid and quenching thirst. Studies have shown that puerarin extracted from puerarin can inhibit the expression of connective tissue growth factor mRNA, thereby blocking the progression of kidney fibrosis; reducing urine albumin, improving glucose metabolism and renal function.

\subsection{Coptis}

Coptis, its main active ingredient is berberine, berberine can reduce blood pressure and blood sugar levels in patients with diabetic nephropathy, and reduce insulin resistance in patients to play a role.

\section{DKD common compound treatment}

\subsection{Liuwei Dihuang Wan}

Liuwei Dihuang Wan is a classic prescription for the treatment of kidney yin loss. Jiuxiang Wang ${ }^{[5]}$ gave Liuwei Dihuang Pills combined with Losartan Potassium to 50 patients with DKD for eight weeks. Compared with the simple Losartan Potassium treatment group, they found that urine microprotein and CRP were improved. 


\subsection{Xuefu Zhuyu decoction}

Xuefu Zhuyu decoction was created by Qingren Wang, a physician in the Qing Dynasty, and it was added or subtracted from Taohong Siwu Decoction and Sini Powder. Bingyi Jiang ${ }^{[6]}$ observed 43 cases of DKD patients and gave the observation group conventional treatment combined with Xuefu Zhuyu Decoction. Compared with the conventional treatment group, they found that $24 \mathrm{~h}$ urine protein quantification and urine protein excretion rate were improved, and they concluded that: The auxiliary treatment of Xuefu Zhuyu Decoction during the treatment of DKD can achieve the effect of delaying the progression of the disease.

\subsection{Jinkui Shenqi Pill Zhang Linghong}

Jinkui Shenqi Pill Zhang Linghong ${ }^{[7]}$ and others observed 82 DKD patients using Jinkui Shenqi Pill. Compared with the conventional Western medicine treatment in the control group, they found that the renal function of the observation group improved better, which shows that this prescription has clinical significance.

\section{Discussion and outlook}

In the prevention and treatment of DKD, many doctors focus on replenishing kidney qi, promoting blood circulation and removing blood stasis. It is an inevitable trend for DKD to enter the collaterals for a long time. The treatment of DKD through a variety of Chinese medicine therapies is more prominent than the treatment of Western medicine, but at the same time, there are many shortcomings in the treatment of Chinese medicine. For example: for the diagnosis and treatment of TCM clinical diseases, this is not good for TCM treatment of DKD. In order to further exert the advantages of TCM therapy in the treatment of DKD, we should strengthen the exploration of TCM clinical differentiation and strive for the standardization of single TCM and compound prescriptions, so as to make TCM treatment of DKD more comprehensive and promote TCM treatment of DKD.

\section{Disclosure statement}

The author declares no conflict of interest.

\section{References}

[1] Chen B, 2011, Discussion on TCM Differentiation and Treatment of Diabetic Nephropathy. Shenyang: Liaoning University of Traditional Chinese Medicine.

[2] Zhang XW, Chen YP, 2008, Chen YP's experience in treating diabetic nephropathy. Shanghai Journal of Traditional Chinese Medicine, 42(6): 6-7.

[3] You LZ, Lin YX, Fang ZH, et al., 2017, Research Progress in the Pharmacological Effects of Astragaloside IV in the Treatment of Diabetes and its Complications. Chinese Journal of Chinese Materia Medica, 42(24): 4700 4706.

[4] Wang QL, Li W, 2016, Research Progress on the Molecular Mechanism of Puerarin in the Treatment of Diabetic Nephropathy. Clinical Research of Traditional Chinese Medicine, 8(4): 40-41, 43.

[5] Wang JX, 2015, Clinical Study of Liuwei Dihuang Pills Combined with Losartan Potassium in the Treatment of Early Diabetic Nephropathy. Modern Medicine and Clinics, 30 (06): 674-677.

[6] Jiang BY, 2014, Observation on the Clinical Effect of Xuefu Zhuyu Decoction in the Treatment of 43 Cases of Diabetic Nephropathy. Integrated Traditional Chinese and Western Medicine and Chinese Medicine, 18(14): 1854-1855.

[7] Zhang LH, 2018, The Effect of Jinkui Shenqi Pills on Diabetic Nephropathy of Spleen and Kidney Yang Deficiency and its Influence on Renal Function. Electronic Journal of Integrated Traditional Chinese and Western Medicine Cardiovascular Diseases, 6(02): 169 -170. 